\title{
Sensor Network with Delay Tolerance (SeNDT)
}

\author{
Paul McDonald Dermot Geraghty Ivor Humphreys \\ Dept. of Mechanical and Manufacturing Engineering \\ Trinity College Dublin \\ pmcdonal@tcd.ie_tgerghty@tcd.ie \\ humphri@tcd.ie
}

\author{
Stephen Farrell Vinny Cahill \\ Department of Computer Science \\ Trinity College Dublin \\ stephen.farrell@cs.tcd.ie \\ vinny.cahill@,cs.tcd.ie
}

\begin{abstract}
As the technology underlying sensor networks becomes more advanced and reliable, ubiquitous computing will come closer to being a reality. This paper details the design and deployment of several sensor nodes that use the delay tolerant network approach in order to meet the relevant application requirements. Two applications are presented, lake water quality monitoring in rural lakes and noise level logging in urban areas and along motorways. The installations are discussed and initial results presented.
\end{abstract}

Keywords: Delay tolerant networking (DTN), lake water quality, environmental noise monitoring, A-weighting.

\section{INTRODUCTION}

Sensor network research has, in recent years, become one of the hot topics in both academia and industry. Vast amounts of money and resources have been dedicated to designing smarter, cheaper and more efficient ways of observing the world around us. The idea of distributed systems of sensors capable of monitoring and interacting with our environment has even become commonplace. Many sensor networking scenarios presented involve dense networks of small, cheap, very low power nodes. But how much of this research is focused on purely theoretical issues and the "what if this were the case" approach? Simulations can only provide so much useful data. Optimisation without realisation will only ever yield limited progress.

Conventions, such as they are in this relatively new field, tend mainly toward concentrations of low powered microsensors which aggregate data to form an overall picture of a larger area. Limited communication ability necessitates large scale deployments in order to ensure reliable delivery of data to the destination [1].

Dept. Mechanical Engineering, Trinity College Dublin, Ireland. Email: pmcdonal@tcd.ie
Such large scale deployments bring with them higher costs in terms of equipment, deployment costs and, a factor often ignored, recovery costs.

The approach presented in this paper deviates somewhat from this trend by deploying a relatively high power sensor network, when compared to say, motes [2]. The increased power consumption and cost are justified by increased sensing and communication ranges and an overall cheaper and more robust network.

\subsection{Delay Tolerant Networking and SeNDT}

A delay tolerant network (DTN) is an overlay network that operates on top of several regional networks. The purpose of a DTN is to facilitate interoperability between these networks, some of which are challenged by limitations such as limited connectivity, long or variable delays, asymmetric data rates or high error rates [3]. By using DTN protocols such as the Bundle Protocol (BP) or Licklider Transmission Protocol (LTP), reliable communication and dissemination of data can be achieved in situations where conventional networks would fail [4].

Originally driven by the need for reliable deep space communications, the DTN technology has several terrestrial applications, such as sensor networks operating in harsh or remote locations.

The SeNDT (Sensor Network with Delay Tolerance) group $^{1}$ at Trinity College Dublin have developed a versatile and robust sensor platform, designed to be deployed for long periods of time in harsh environments. Our pilot study application involves water quality monitoring in rural Ireland and environmental noise monitoring in urban areas and on national motorways. Our initial pilot study was deployed with the help of Dublin City Council and the Irish National Roads Authority. This study has provided valuable end-user feedback to the design team. In addition, installing the units has brought to light some of the practicalities involved in moving from a design scenario to actually implementing a real world, operational sensor network.

\footnotetext{
${ }^{1}$ https://down.dsg.cs.tcd.ie/sendt
} 


\subsection{Hardware}

The SeNDT node is a general purpose hardware platform running a Linux based operating system. The hardware is split into three parts, the processor board, the main $\mathrm{I} / \mathrm{O}$ board and the expansion board.

The hardware has a range of interfaces both for sensors and communication links. These include a high performance analog front end, host and device controller USB ports and multiple RS232 ports. Data is stored on a compact flash card, with up to 4GB of memory. In addition the processor board has $32 \mathrm{MB}$ of on-board flash memory. The processor is an Intel PXA255 with 64MB of SDRAM and DSP (Digital Signal Processing) extensions, which are useful for real time processing of audio signals. The main communications interface is over an 802.11 b wireless link, via a PCMCIA card. Solar charging is made possible by power control circuitry which regulates charging and monitors battery levels. This enables operation in locations where no other power source is available. While the device is not inherently low powered, it can be operated on a power saving schedule.

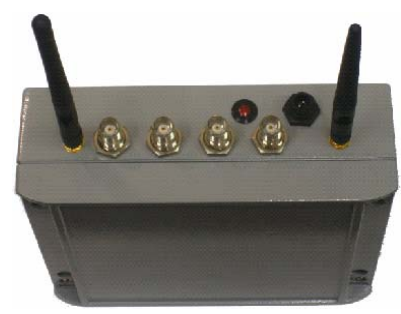

Figure 1: A SeNDT Noise Monitoring Unit

\subsection{SOFTWARE}

SeNDT nodes run under the Linux operating system (2.6.15 kernel arm-linux build). The main advantage of this is that we can use standard tools to communicate with and configure the units [5]. The lake units operate on roughly a $10 \%$ duty cycle. Schedules are centrally generated and stored in XML format ${ }^{2}$, the binary equivalent is stored on each unit. Communications are handled using the Trinity College Dublin LTP implementation ${ }^{3}$ running over UDP (User Datagram Protocol) [5]. Upon waking the nodes run a diagnostic program which logs the battery levels, and communications activity.

For the noise monitoring application we acquire the data from the microphones (up to 4), calculate the noise levels and then $\log$ this data to the compact flash. The acquired data is A-weighted. This is a filter which normalises the sound levels relative to the response of the

\footnotetext{
${ }^{2}$ The XML Schema for our current schedule format is available at: https://down.dsg.cs.tcd.ie/schemas/

${ }^{3}$ Our LTP library code is available from

https://down.dsg.cs.tcd.ie/ltplib/
}

human ear. The RMS sound pressure level is then calculated and from this standard sound averaging values such as Leq, L10 and L95 are found.

\section{Application Rationale}

\subsection{Lake Water Quality Monitoring}

Lake water quality in Irish lakes has been declining steadily in recent years. This is largely due to run off from farms and industrial sites. Increases in the amount of phosphates and nitrates in the water leads to eutrophication of lakes. Eutrophication (over-enrichment with nutrients) itself is not necessarily harmful, but it can cause changes to the ecological balance of a water system, which may interfere with human activities and reduce biodiversity. An example is the decline of the fish population, which has a knock-on effect on local tourism. It is because of these consequential effects that eutrophication is often undesirable and needs to be controlled or reversed [6].

Current water quality monitoring practices involve taking periodic samples at specific locations and analysing the samples in a laboratory. A reliable, in situ system for monitoring changes in water quality is a requirement if any action is to succeed in tracking and combating water pollution.

\subsection{Noise Level Monitoring}

The European Union have published proposals to establish a common EU framework for the assessment and management of exposure to environmental noise. The goal of the Directive is to preserve a "high level of health and environmental protection" and to harmonise noise indicators and assessment methods. Under the Directive, member states must produce noise maps for all major cities and roads by June 2007 [7].

An environmental noise map is a graphical representation of the noise levels in a particular area with different colours representing variations in average noise values in $\mathrm{dB}$. The common indicator is $\mathrm{L}_{d e n}$, a daily average, weighted for day, evening and night time readings. Similar to how isobars link areas of equal pressure on a weather map, the contours on a noise map (isophons) join points which have the same noise level. Noise maps can be linked to population distribution information to determine how many people are affected by a certain source of noise. Subsequently the maps can be used to measure the effectiveness of preventative measures, such as re-routing of traffic or the placement of noise barriers. Noise maps will prove to be valuable tools when it comes to issues such as planning new roads or industries in addition to the creation of noise action plans. A sample noise map of Trinity College is shown in Figure 2. 


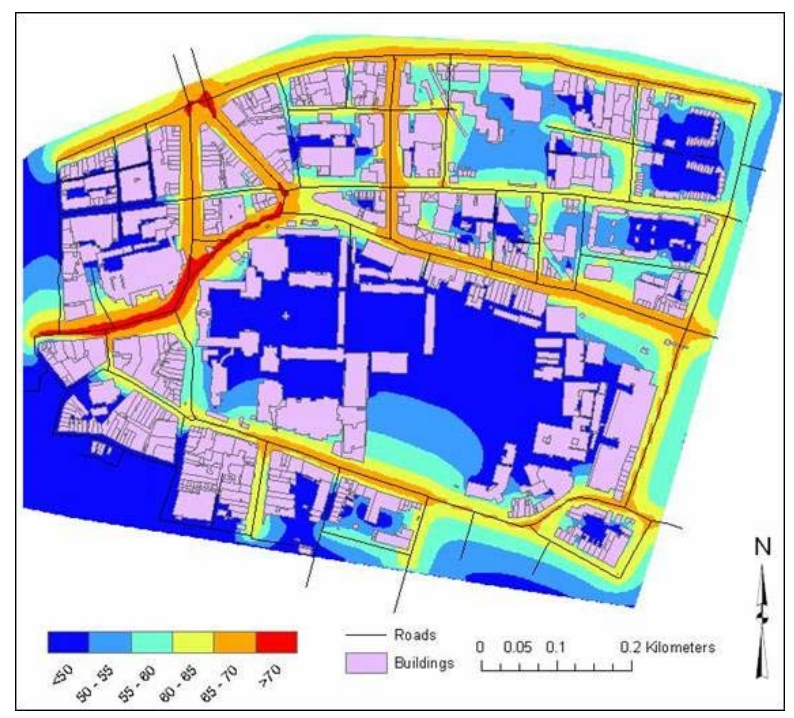

Figure 2: Night-time average noise emissions $\left(L_{\text {night }}\right)$ in decibels (dBA) [8]

While the maps are originally generated using predictive software, the levels must be calibrated and corrected with actual readings. To accurately assess noise levels over time it is necessary to implement a cost effective system capable of constantly monitoring for extended periods.

\section{DEPLOYMENT AND INSTALLATION}

\subsection{Lake Water Monitoring}

For deployment in such remote locations SeNDT units must be able to overcome the challenges involved with operating for extended periods with limited power. Our power management circuitry controls the interface to a smart battery, similar to those used in laptops, and regulates battery charging from our solar panel. Two high gain external antennas were connected to the wireless card to increase radio range.

The SeNDT boards are enclosed within an IP67 aluminium enclosure. All cables are potted into a bulkhead union, which is sealed both inside and out with silicon sealant. This assembly is mounted on a standard 18 " ring buoy. An 11Watt marine grade flexible solar panel (Unisolar FLX-11) is bent over the enclosure and attached to the buoy (see figures $3 \& 4$ ). A small weight attached to the bottom of the buoy acts as ballast and the entire arrangement is chained to an anchor.

The units were deployed in a large lake near Killeshandra Co. Cavan, an area that has suffered badly from water pollution. This has serious implications for

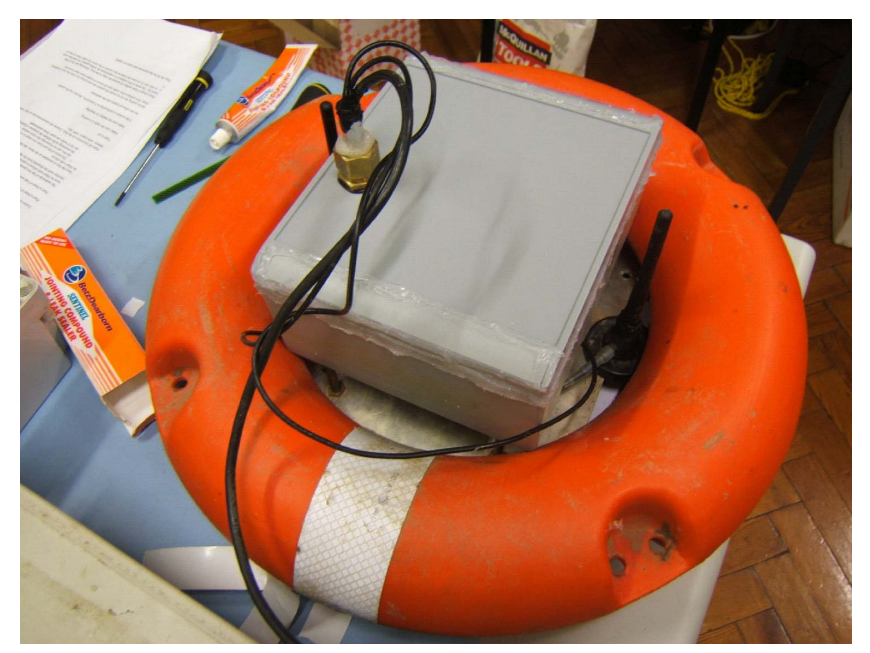

Figure 3: A sealed lake node

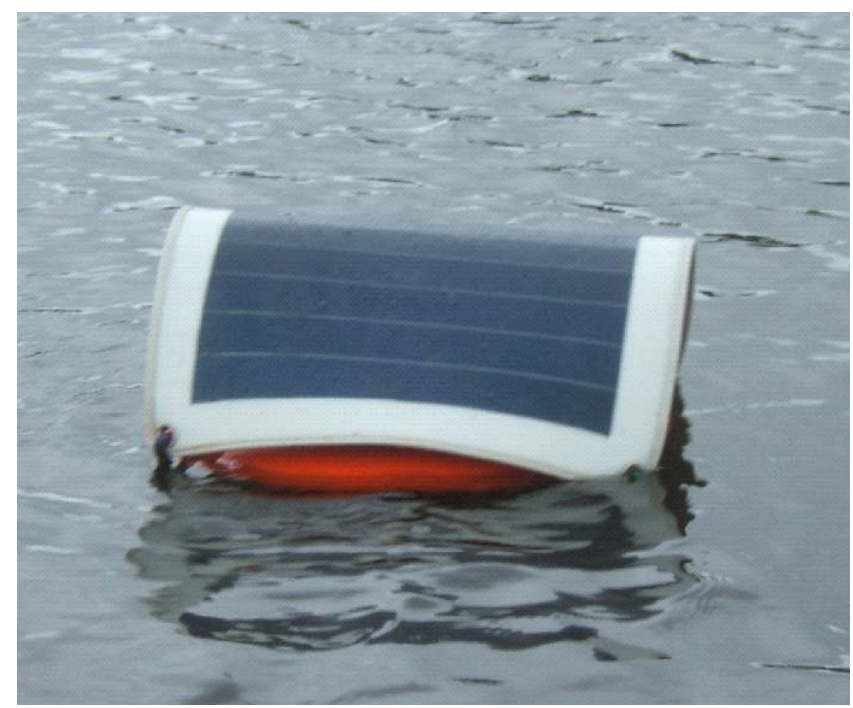

Figure 4: A node in the lake

angling and tourism in the local area. A decline in the fish population will have a major effect on local industry, most of which is reliant on tourist fishermen. Once deployed the nodes were left in place for approximately one month at a time (so far).

Our goal was to assess the suitability of these particular solar panels and properly determine our power requirements. While the overall vision of the project is to deploy a number of units all monitoring pertinent water quality parameters, the cost of suitable sensors has so far prohibited us from properly instrumenting our nodes (approx. $€ 6,000$ per unit). Before such capital outlay can be justified, the network itself must be proved and the longevity of the nodes assured. This latter aspect is, as with many sensor network deployments, still a work-in-progress.

\footnotetext{
${ }^{4} \mathrm{IP}$ - Ingress Protection rating. The first digit refers to protection against the ingress of solid matter (1-6), the second to liquid (1-8)
} 


\subsection{Noise Monitoring}

Locations for deployment were chosen by our pilot study partners. Two urban sites were selected by Dublin City Council, and a further five were chosen by the National Roads Authority, on main motorways around Ireland.

The units were each equipped with four low cost electret microphones, attached to our $\mathrm{A} / \mathrm{D}$ converter and housed within protective coverings.

The urban sites were at inner city locations, on the Dublin Quays. This is an area that experiences high volumes of commercial traffic, consisting mainly of large trucks en route to the Dublin docks. The nodes were placed in secure enclosures mounted on traffic light poles (see figure 5).

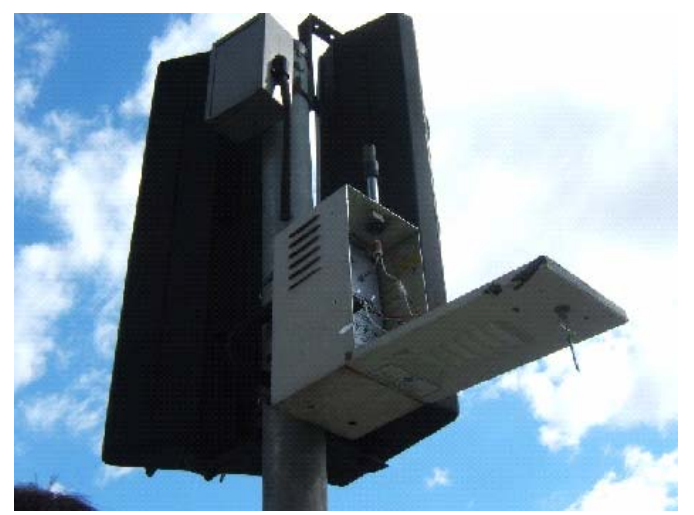

Figure 5: An urban noise monitoring installation site

The motorway locations chosen were at sites where existing traffic counting units were housed in small steel cabinets by the roadside. The idea being to correlate noise level data with traffic counter readings. In order to limit exposure we mounted the microphones on the side of the cabinet, but kept all wires and antennas inside (figure 6). This resulted in attenuation of radio signals and a reduction in communication range. While this is not an issue where manual data collection is involved, it may impact the performance of future automated data collection by instrumented vehicles. (We may have to move to using patch antennae mounted externally in future.)

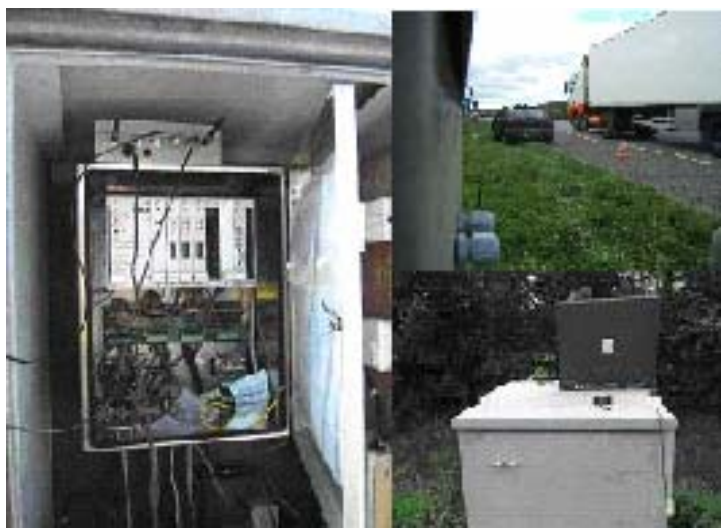

Figure 6: A motorway noise monitoring site

\subsection{Configuration}

The lake units were scheduled to wake for two minutes every half hour. It takes approximately 40 seconds to complete the boot cycle, then the results of diagnostic functions were logged at successive 40 second intervals. After 2 minutes an RTC (Real Time Clock) controlled shut down was executed.

The noise units were configured to run on a one hour sampling loop, logging noise levels every 5 minutes. Standard noise analysis techniques evaluate either 5 minute, 15 minute or 1 hour time slots. Our configuration allowed for simple data processing using any of these reference periods.

\subsection{End-User Interface}

Ease of use for the end user was an issue that we had not previously addressed until the pilot study. Often prototype or development technologies require the user to have a relatively high level of technical knowledge. Although we were using commonplace Wi-Fi communications, actually using the devices required a good working knowledge of the Linux command line interface. Rather than asking potential users (and potential customers) to become familiar with Linux we decided to use a GUI interface. WinSCP [9] is an open source FTP/SSH client for Windows. It uses SSH and supports the legacy SCP protocol. Its Windows Explorer style display manages secure file transfers between remote and local machines with drag-and-drop functionality. This was ideal for our purposes as it allowed the end users take responsibility for data collection without requiring any specialised expertise.

\section{PERFORMANCE AND RESUlts}

Currently all data collection for both pilot studies is done manually. In the case of the lake this is facilitated by placing the nodes close enough to the shore to receive a signal (approx. 100m). The noise monitoring data is collected by staff from DCC and the NRA periodically. Our goal however, is to fully automate both situations. This will be done with the use of data mules [10], a concept present in many DTNs. A data mule is essentially a mobile node that can route data between stationary nodes and a data sink.

\subsection{Lake Water Monitoring}

The lake pilot study has produced mixed results so far. The duty cycle chosen provided for reasonable granularity in terms of water quality monitoring. Sampling once or twice an hour would provide much greater resolution than manually taking samples once every few weeks. With this in mind it was agreed that the recharging capabilities should be able to provide for this mode of operation, rather 
than simply diminishing duty cycles until a balance was found. Although the latter approach might eventually yield a working configuration, the goal was to determine if our solar panel was up to the task of running our unit under our desired operating conditions.

Deployments done early in the summer of 2006 showed favourable charging conditions when operating normally. Average daily charging either matched or exceeded power consumption (see figure 7).

However, further deployments done in early winter showed different results. The amount of average daily sunshine had dropped dramatically. A reduction in daylight hours and deterioration in general weather conditions meant that we did not charge at all during this period (see figure 8). It is clear that weather conditions in winter provide a serious challenge to our installations in terms of power management.

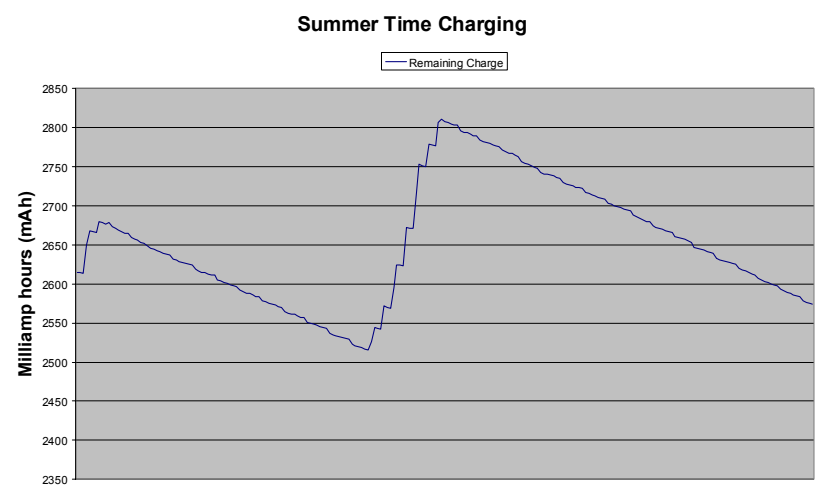

Figure 7: Charge characteristic over a 48 hour period (summer)

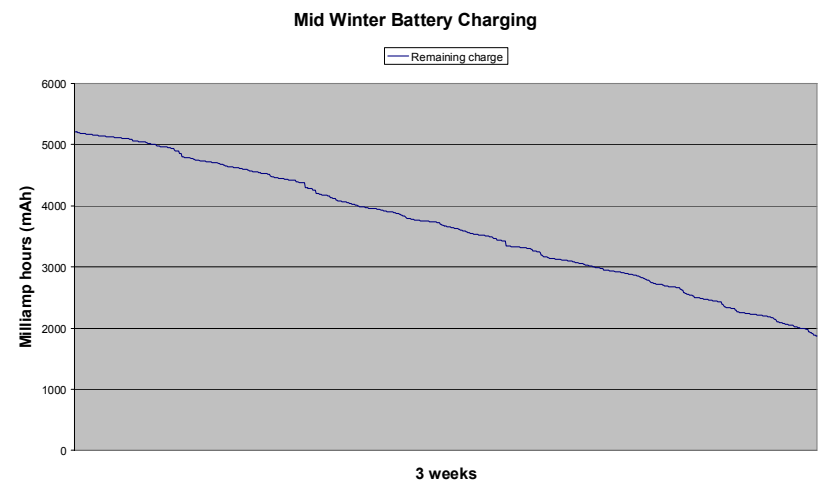

Figure 8: Discharge characteristic over 3 weeks (winter)

\subsection{Noise Level Monitoring}

Initial results from the noise monitoring pilot study have been promising. Performance problems were identified and addressed very early on. The physical make up of the prototype proved to be a highly efficient design. The units were well protected and the microphones weathered very well, even in harsh winter conditions.

The noise data itself is also encouraging. Noise levels seen so far match expected trends. Sound levels followed a diurnal pattern, fluctuating between approximately 50dBA and $100 \mathrm{dBA}$. This was close to expected noise levels for this type of urban location. As can be seen from the data sets in figures 9-11 there is an obvious cyclic drop in noise levels during the night period, which is expected following an investigation of average Dublin traffic counts.

Noise level measurements:

Leq: essentially the average noise level

L10: level exceeded $10 \%$ of the time

L95: level exceeded 95\% of the time (background noise)

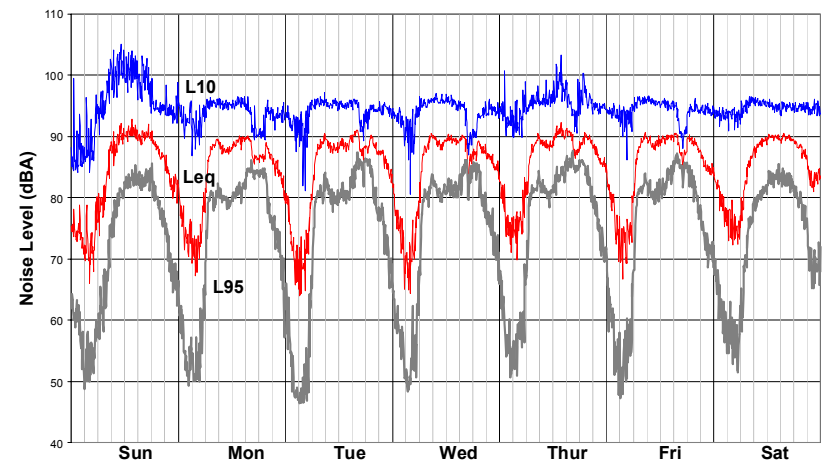

Figure 9: Motorway noise levels over a week long period

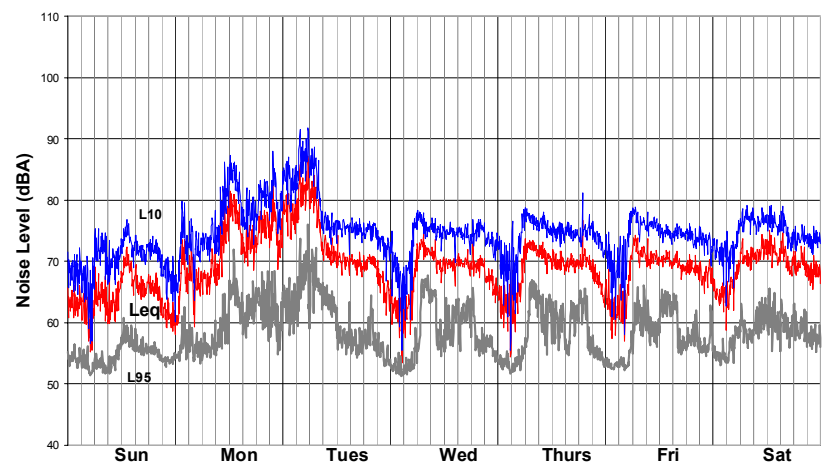

Figure 10: Urban noise levels over a week long period 


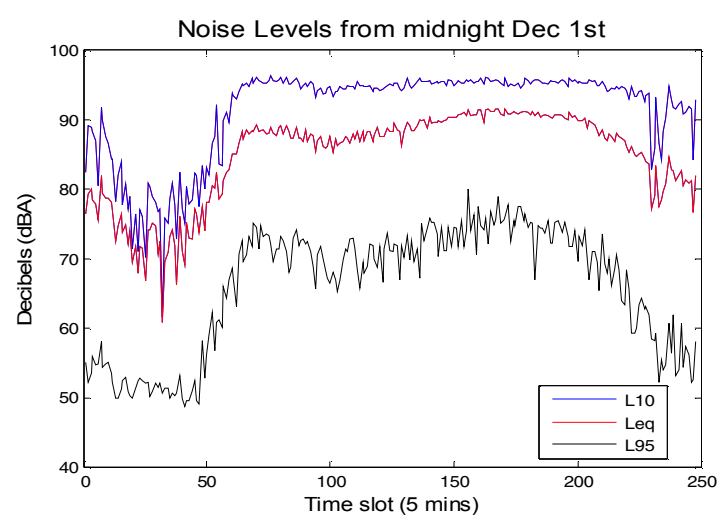

Figure 11: Motorway noise levels over a single day

\section{Discussion \& Future Work}

Distributed sensor systems hold great potential for improving our understanding of the world around us. Whether they are delicate microsensors for medical applications or rugged nodes for deployment in harsh environments, these systems will no doubt become more and more widespread as time goes on. But only by actually deploying these systems in real world applications will we truly be able to learn how to best implement this technology.

This paper presents the design and deployment of SeNDT nodes. These nodes are highly versatile and rugged sensor platforms, suitable for a variety of sensing applications.

Initial results have shown that the power requirements for remote deployments may present a challenge in winter conditions. We plan to test more powerful or efficient solar panels and also to re-evaluate our power budget to incorporate context aware power management.

The noise pilot study has proved very successful and feedback from our pilot study partners has allowed us to refine the design and performance of the units. Early results are promising and further improvements will be made over time.

Once the installations are completed, the next major task is to take advantage of the delay tolerant communication capabilities of our nodes. This will be of great benefit in terms of automated data collection using data mules. By instrumenting mules and determining optimal communications schedules we can implement a fully automated, delay tolerant, distributed system.

In the case of the lake we plan to install a node in a fishing boat used by local fishermen. The mule node will collect data as it travels throughout the lake and then deliver it to a data sink when it returns to shore.

For the noise monitoring applications we plan to instrument refuse trucks and freight vehicles as data mules. These trucks follow a set route at known times, so communication scheduling is relatively simple. Again the data will be delivered to a data sink when the trucks arrive back at the depot. This database will be linked to an online GIS system capable of plotting noise maps from measured data. By linking actual measurements with existing noise modelling software the SeNDT units will fulfil all relevant requirements as detailed in the European Noise Directive.

\section{CONCLUSIONS}

Environmental monitoring is now becoming an obligatory practice for all national and local authorities in Ireland and throughout Europe. Driven by both legislation and public sentiment, improvements in this field are not only desired but also legally necessary. However, to date no practical measures are in place in Ireland to address these issues.

In this paper we present SeNDT units. These are an effective and versatile solution to the challenges associated with widespread sensing applications. By developing technology to constantly monitor environmental parameters we are responding to a problem that has not previously been tackled in this country.

Our water quality and noise monitoring sensor networks offer a new way to meet the greener requirements of recent EU laws. By operating using DTN protocols we can ensure reliable automated data collection. This is a necessary step if any full scale deployments of environmental monitoring networks are to succeed in useful and practical application scenarios.

\section{REFERENCES}

1. Rex Min, Manish Bhardwaj, Seong-Hwan Cho, Eugene Shih, Amit Sinha, Alice Wang, Anantha Chandrakasan, "Low-Power Wireless Sensor Networks," vlsid, p. 205, The 14th International Conference on VLSI Design (VLSID '01), 2001

2. UC Berkeley Motes (MICA2 and MICA2Dot)

3. Forrest Warthman, "Delay-Tolerant Networks (DTNs)," Warthman Associates, Version 1.1, May 2003

4. S. Farrell, V. Cahill, D. Geraghty, I. Humphreys, and P. McDonald, "When TCP Breaks: Delay- and DisruptionTolerant Networking." IEEE Internet Computing, vol. 10 , no. 4, 2006, pp. 72-78

5 S. Farrell, V. Cahill. "Delay and Disruption Tolerant Networking," ISBN 1-59693-063-2, Artech House, 2006

6. J. Keyes, F. Gibbons, Envionmental Section, Cavan County Council, "The Quality of River and Lake Water in County Cavan," Report for the year 2004, April 2005

7. The European Parliament and Council of the European Union, "Directive 2002/49/EC of the European Parliament and of the Council," Official Journal of European Communities, 2002

8. Eoin King, www.mme.tcd.ie/ eoin, Department of Mechanical Engineering, Trinity College Dublin, 2007

9. Martin Prikryl, http://winscp.net

10. R. C. Shah, S. Roy, S. Jain, W. Brunnette, "Data Mules: Modeling a three-tier Architecture for Sparse Sensor Networks,” Intel Research, IRS-TR-03-001, 2003 